\title{
NCOA4-Mediated Ferritinophagy: A Vicious Culprit in COVID-19 Pathogenesis?
}

\author{
Fengju Jia ${ }^{1 *}$, Hongxia Liu $^{2}$ and Shan Kang ${ }^{3}$ \\ ${ }^{1}$ School of Nursing, Qingdao University, Qingdao, China, ${ }^{2}$ Yantai Ludong Hospital (Shandong Provincial Hospital Group), Yantai, \\ China, ${ }^{3}$ Department of Laboratory, Qingdao Eighth People's Hospital, Qingdao, China
}

Coronavirus disease 2019 (COVID-19) is a global pandemic that has caused widespread loss of life. Notably, in this disease, severe inflammatory reactions characterized by cytokine storms are caused by severe acute respiratory syndrome coronavirus 2 . The cytokine storms may promote hyper-ferritinemia which can further intensify the inflammation. Moreover, elevated ferritin levels trigger nuclear receptor coactivator 4 (NCOA4)-mediated ferritinophagy, in which ferritin is degraded and iron is released. Excess iron released from ferritinophagy can promote ferroptosis and cellular damage. Therefore, we propose that NCOA4-mediated ferritinophagy can be targeted to limit the ferroptosis and prevent the multi-organ damage and severity in COVID-19 patients.

OPEN ACCESS

Edited by:

Binod Kumar,

Loyola University Chicago,

United States

Reviewed by:

Sanjay Pandey,

Albert Einstein College of Medicine,

United States

*Correspondence:

Fengju Jia

jiafengjv@163.com

Specialty section:

This article was submitted to

Molecular Diagnostics and

Therapeutics,

a section of the journal

Frontiers in Molecular Biosciences

Received: 20 August 2021 Accepted: 30 November 2021

Published: 15 December 2021

Citation:

Jia F, Liu H and Kang S (2021) NCOA4-Mediated Ferritinophagy: A Vicious Culprit in COVID-19

Pathogenesis?

Front. Mol. Biosci. 8:761793.

doi: $10.3389 /$ fmolb.2021.761793
Keywords: ferritinophagy, NCOA4, hyperferritinemia, iron, COVID-19

\section{INTRODUCTION}

Coronavirus disease 2019 (COVID-19), triggered by the novel severe acute respiratory syndrome coronavirus 2 (SARS-CoV-2), is a serious health concern and can be profoundly detrimental. In March 2020, the World Health Organization declared it as a pandemic. Although various vaccines have been developed, only limited number of therapeutic drugs is available. A significant proportion of patients with COVID-19 experience severe interstitial pneumonia, possibly resulting in acute respiratory distress syndrome and systemic inflammatory response syndrome.

\section{COVID-19 Systemic Inflammatory Response}

COVID-19 systemic inflammatory reaction can be life-threatening due to its hyper-inflammation sustained by a cytokine storm. The cytokine storm is not only one of the earliest and most debilitating symptoms in patients with COVID-19 but also contributes to the disease severity at the later stage (Siddiqi and Mehra, 2020). Once inside the cells, the SARS-CoV-2 RNA is recognized by the pathogen recognition receptors, which trigger a downstream cascade of molecules leading to the activation of transcription factors, such as nuclear factor kappa B and interferon regulatory factor 3 (IRF-3) and the subsequent production of type 1 interferons and several other pro-inflammatory cytokines (e.g., interleukin [IL]-1 $\beta$ and IL-6) (Kawai and Akira, 2010; Conti et al., 2020; Yang et al., 2020). Considerably, elevated plasma levels of plasma pro-inflammatory cytokines, such as IL-6, IL2, IL-7, IL-10, IFN- $\gamma$-induced protein 10 (IP-10), monocyte chemoattractant protein-1 (MCP-1), and macrophage inflammatory protein-1 alpha (MIP-1a), have been observed in some patients (Han et al., 2020; Karki et al., 2021). This expansion of an uncontrolled inflammatory response due to SARS-CoV-2 infection potentially leads to cell death by apoptosis, necrosis, or ferroptosis (a newly discovered type of programmed cell death), resulting in multi-organ damage in patients with COVID-19 (Banchini et al., 2021). Considering most of the acute and chronic deleterious effects of 
the SARS-CoV-2 infection are the consequences of hyperinflammatory response of the virus, several inflammatory drugs have been used for the treatment of severe COVID-19 (Zhang et al., 2020).

\section{HYPER-FERRITINEMIA IN COVID-19: A PART OF HYPER-FERRITINEMIC SYNDROME SPECTRUM}

COVID-19-associated systemic inflammation is thought to be the part of the hyper-ferritinemic syndromes. COVID-19 shares many clinical and laboratory features such as lymphopenia, reduced NK cell numbers and activity, abnormal liver functions, and coagulopathy, with the other hyper-ferritinemic syndromes such as macrophage activation syndrome (MAS) and adult-onset Still's disease (AOSD) (Rosario et al., 2013; Shoenfeld, 2020). Elevated pro-inflammatory cytokine and ferritin levels are the other hallmarks of hyper-ferritinemic syndromes common to COVID-19 (Colafrancesco et al., 2020).

Ferritin levels are elevated in various inflammatory disorders and directly correlated with poor prognosis and severity of the COVID-19 patients (Mehta et al., 2020). Although the source of circulating serum ferritin in COVID19 has not yet been determined, macrophages contribute considerably to release ferritin via a non-classical secretory pathway (Cohen et al., 2010). A recent in vitro study showed that hepatocytes could actively secrete ferritin (Ghosh et al., 2004). Besides its active secretion in hepatocytes, serum ferritin is also derived by hepatic cell death (Colafrancesco et al., 2020). Furthermore, a key iron-regulatory hormone hepcidin could increase intracellular ferritin levels by sequestering iron in enterocytes and macrophages (Daher et al., 2017). Activated innate immunity and cytokine cascades can accelerate the expression of hepcidin (Drakesmith and Prentice, 2012; Cassat and Skaar, 2013). Interestingly, SARS-CoV-2 can mimic hepcidin by inducing ferroportin blockage and can result in the elevated ferritin levels independent of the inflammatory response (Cavezzi et al., 2020). Overall, patients with COVID-19 experience hyper-ferritinemia through the above mechanism.

\section{HYPER-FERRITINEMIA AMPLIFIES INFLAMMATION AND THE CYTOKINE STORM}

The magnitude of the cytokine storm has a positive correlation with the serum ferritin levels. The high circulating ferritin levels in COVID-19 may not only reflect an acute phase response but also display other immunomodulatory functions (Recalcati et al., 2008; Edeas et al., 2020). Ferritin contains two subunits: L and H. $\mathrm{H}$ ferritin induces the expression of inflammatory cytokines such as IL-beta and IL-6 and promotes the myeloid and lymphocyte proliferation by stimulating a specific ferritin receptor TIM-2 (Ruscitti et al., 2016). Also, there is compelling evidence that the cytokine storm in hepatic cells is directly correlated with the extracellular ferritin levels (Ruddell et al., 2009). Thus, the mutual promotion of ferritin and inflammatory cytokine levels generates a vicious loop that constantly heightens the inflammatory state.

\section{IRON DYSHOMEOSTASIS IN COVID-19}

Ferritin is composed of 24 subunits, which can bind up to 4,500 atoms of iron. This makes ferritin one of the major iron storage proteins in the cells. It incorporates $\mathrm{Fe}^{2+}$ via ferritin iron pores and further oxidizes $\mathrm{Fe}^{2+}$ to $\mathrm{Fe}^{3+}$ by an $\mathrm{H}$ subunit inside the ferritin cage, leading to inert deposits of $\mathrm{Fe}^{3+}$ that are unavailable for intracellular use or generation of reactive oxygen species (ROS) (Philpott, 2018). Ferritin can also release $\mathrm{Fe}^{3+}$ and reduce it to $\mathrm{Fe}^{2+}$ (Kidane et al., 2006), indicating that iron is critical in COVID-19 pathogenesis (Bellmann-Weiler et al., 2020; Edeas et al., 2020). Notably, iron overload directly influences several critical manifestations of COVID-19 such as systemic inflammation, hyper-ferritinemia, and immune dysfunction.

COVID-19-associated iron overload can be triggered by the release of the free iron from the damaged hemoglobin and ferritin catabolism. The process of viral infection is facilitated by several SARS-CoV-2 accessory viral proteins, including open reading frames (ORFs)-3, 10, and 8. The virus can interact with hemoglobin through angiotensin-converting enzyme 2 (ACE2 ), CD-147, CD-26, and other receptors. The heme on the 1-beta chain of hemoglobin is attacked by ORF-8 and surface glycoprotein binding to porphyrin. Consequently, hemolysis and dysfunctional hemoglobin are engendered by SARS-CoV-2 infection, with or without forming a complex with the released heme (Cavezzi et al., 2020). Damage to hemoglobin causes dissociation of the porphyrins from iron and releases iron into the circulation resulting in the condition of iron overload (Cavezzi et al., 2020).

A retrospective cohort study showed that ferritin could lose a part of its inner iron content, giving rise to extremely high serum levels of free iron (Pretorius and Kell, 2014). Iron deposits derived from the ferritin lead to a poorly labile iron pool, indicating that the abundance of ferritin is a key factor governing iron homeostasis. Conversely, depletion of ferritin results in the release of iron into the labile iron pool, resulting in increased sensitivity to ferroptosis (Stockwell et al., 2020). Several studies have suggested that the selective autophagic turnover of ferritin (ferritinophagy) contributes to ferroptosis in fibroblasts and cancer cells (Hou et al., 2016). Although the role of ferritinophagy in COVID-19 remains unclear, its contribution in the pathological processes of neurodegeneration, cancer, ischemia/reperfusion injury, and urinary tract infections is well established (Tang et al., 2018).

Iron metabolism is objectively related to clinical syndromes during COVID-19 by inducing a series of biological events (Cavezzi et al., 2020). By interacting with molecular oxygen, excess intracellular iron can generate ROS (through Haber-Weiss and Fenton reactions), reactive nitrogen species (RNS), and reactive sulfur species (RSS) 


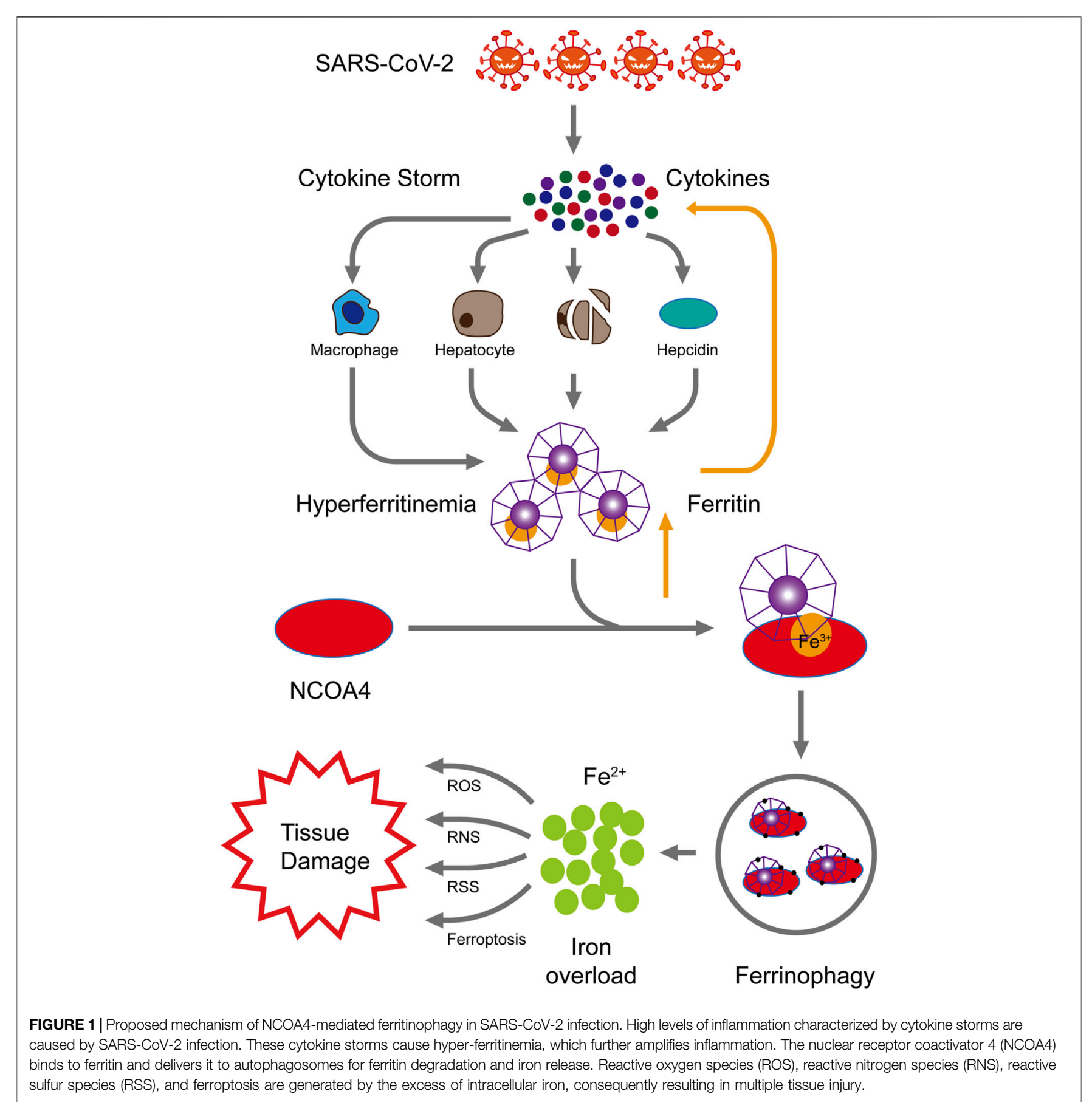

(Oexle et al., 1999). Through redox damage, mitochondrial dysfunction is favored, resulting in ferroptosis, multiple tissue damage, and subsequent fibrosis (Le Lan et al., 2005). During severe inflammatory conditions, excess iron can deteriorate the inflammatory reaction by inducing a severe pro-coagulant state (Pretorius and Kell, 2014). Moreover, higher iron levels may facilitate virus multiplication in the host cells (Drakesmith and Prentice,
2008). Iron depletion or chelation has been considered as a potential antiviral therapy to protect against excessive inflammatory responses and tissue damage by sequestering iron and preventing oxygen radical formation and lipid peroxidation in patients with COVID-19 (Perricone et al., 2020). In addition, the binding of SARS-CoV-2 to its receptors for entry into host cells can be prevented by lactoferrin, an iron chelator (Rainey et al., 2019; Chang et al., 2020). 


\section{NUCLEAR RECEPTOR COACTIVATOR 4 (NCOA4)-MEDIATED FERRITINOPHAGY IN COVID-19}

Recently, a ferroptosis signature has been observed in patients with COVID-19 (Jacobs et al., 2020). Ferroptosis inhibitors, such as ferrostatin-1, have been applied as potential drug candidates for COVID-19. Notably, NCOA4 has been reported to be the cargo receptor of ferritin in ferritinophagy (Dowdle et al., 2014; Mancias et al., 2014). NCOA4 binds to ferritin and delivers it to autophagosomes for ferritin degradation and iron release. ROS, RNS, RSS, and ferroptosis are generated by excess iron, consequently resulting in multiple tissue injury (Supplementary Figure S1). Additionally, Joseph et al. demonstrated that a direct association between a key surface arginine in ferritin and a $\mathrm{C}$-terminal element in NCOA4 is required for the delivery of ferritin to the lysosome via autophagosomes (Mancias et al., 2015). Notably, NCOA4 depletion inhibits the delivery of ferritin to the lysosome, resulting in the disruption of ferritin degradation. The degradation of NCOA4 by HERC2, an E3 ubiquitin ligase, leads to suppressed ferritinophagy and elevated levels of ferritin (Mancias et al., 2015).

Therefore, we hypothesize that cytokine storms caused by SARS-COV-2 infection may promote hyper-ferritinemia which can further intensify the inflammation. Elevated ferritin levels can

\section{REFERENCES}

Banchini, F., Cattaneo, G. M., and Capelli, P. (2021). Serum Ferritin Levels in Inflammation: a Retrospective Comparative Analysis between COVID-19 and Emergency Surgical Non-COVID-19 Patients. World J. Emerg. Surg. 16 (1), 9. doi:10.1186/s13017-021-00354-3

Bellmann-Weiler, R., Lanser, L., Barket, R., Rangger, L., Schapfl, A., Schaber, M., et al. (2020). Prevalence and Predictive Value of Anemia and Dysregulated Iron Homeostasis in Patients with COVID-19 Infection. Jcm 9 (8), 2429. doi: $10.3390 /$ jcm 9082429

Cassat, J. E., and Skaar, E. P. (2013). Iron in Infection and Immunity. Cell Host Microbe 13 (5), 509-519. doi:10.1016/j.chom.2013.04.010

Cavezzi, A., Troiani, E., and Corrao, S. (2020). COVID-19: Hemoglobin, Iron, and Hypoxia beyond Inflammation. A Narrative Review. Clin. Pract. 10 (2), 1271. doi: $10.4081 /$ cp. 2020.1271

Chang, R., Ng, T. B., and Sun, W.-Z. (2020). Lactoferrin as Potential Preventative and Adjunct Treatment for COVID-19. Int. J. Antimicrob. Agents 56 (3), 106118. doi:10.1016/j.ijantimicag.2020.106118

Cohen, L. A., Gutierrez, L., Weiss, A., Leichtmann-Bardoogo, Y., Zhang, D.-l., Crooks, D. R., et al. (2010). Serum Ferritin Is Derived Primarily from Macrophages through a Nonclassical Secretory Pathway. Blood 116 (9), 1574-1584. doi:10.1182/blood-2009-11-253815

Colafrancesco, S., Alessandri, C., Conti, F., and Priori, R. (2020). COVID-19 Gone Bad: A New Character in the Spectrum of the Hyperferritinemic Syndrome? Autoimmun. Rev. 19 (7), 102573. doi:10.1016/j.autrev.2020.102573

Conti, P., Ronconi, G., Caraffa, A., Gallenga, C. E., Ross, R., Frydas, I., et al. (2020). Induction of Pro-inflammatory Cytokines (IL-1 and IL-6) and Lung Inflammation by Coronavirus-19 (COVI-19 or SARS-CoV-2): Anti-inflammatory Strategies. J. Biol. Regul. Homeost Agents 34 (2), 327-331. doi:10.23812/CONTI-E

Daher, R., Manceau, H., and Karim, Z. (2017). Iron Metabolism and the Role of the Iron-Regulating Hormone Hepcidin in Health and Disease. Presse Med. 46 (12 Pt 2), e272-e278. doi:10.1016/j.lpm.2017.10.006 trigger NCOA4-mediated ferritinophagy and may lead to ferroptosis, cell death, and organ damage. NCOA4-mediated ferritinophagy can be targeted to limit the ferroptosis and, therefore, prevent the multi-organ damage and severity in COVID-19 patients (Figure 1). Further studies should be conducted to confirm the involvement of NCOA4 and ferritinophagy in SARS-CoV-2 infection.

\section{AUTHOR CONTRIBUTIONS}

The first draft of the manuscript was written by FJ. SK and HL commented on previous versions of the manuscript.

\section{FUNDING}

This work was supported by grants from the Shandong Provincial Natural Science Foundation (ZR2020QC088).

\section{SUPPLEMENTARY MATERIAL}

The Supplementary Material for this article can be found online at: https://www.frontiersin.org/articles/10.3389/fmolb.2021.761793/ full\#supplementary-material

Dowdle, W. E., Nyfeler, B., Nagel, J., Elling, R. A., Liu, S., Triantafellow, E., et al. (2014). Selective VPS34 Inhibitor Blocks Autophagy and Uncovers a Role for NCOA4 in Ferritin Degradation and Iron Homeostasis In Vivo. Nat. Cel Biol 16 (11), 1069-1079. doi:10.1038/ncb3053

Drakesmith, H., and Prentice, A. M. (2012). Hepcidin and the Iron-Infection Axis. Science 338 (6108), 768-772. doi:10.1126/science.1224577

Drakesmith, H., and Prentice, A. (2008). Viral Infection and Iron Metabolism. Nat. Rev. Microbiol. 6 (7), 541-552. doi:10.1038/nrmicro1930

Edeas, M., Saleh, J., and Peyssonnaux, C. (2020). Iron: Innocent Bystander or Vicious Culprit in COVID-19 Pathogenesis? Int. J. Infect. Dis. 97, 303-305. doi:10.1016/j.ijid.2020.05.110

Ghosh, S., Hevi, S., and Chuck, S. L. (2004). Regulated Secretion of Glycosylated Human Ferritin from Hepatocytes. Blood 103 (6), 2369-2376. doi:10.1182/ blood-2003-09-3050

Han, H., Ma, Q., Li, C., Liu, R., Zhao, L., Wang, W., et al. (2020). Profiling Serum Cytokines in COVID-19 Patients Reveals IL-6 and IL-10 Are Disease Severity Predictors. Emerging Microbes \& Infections 9 (1), 1123-1130. doi:10.1080/ 22221751.2020.1770129

Hou, W., Xie, Y., Song, X., Sun, X., Lotze, M. T., Zeh, H. J., 3rd, et al. (2016). Autophagy Promotes Ferroptosis by Degradation of Ferritin. Autophagy 12 (8), 1425-1428. doi:10.1080/15548627.2016.1187366

Jacobs, W., Lammens, M., Kerckhofs, A., Voets, E., Van San, E., Van Coillie, S. et al. (2020). Fatal Lymphocytic Cardiac Damage in Coronavirus Disease 2019 (COVID-19): Autopsy Reveals a Ferroptosis Signature. ESC Heart Fail. 7 (6), 3772-3781. doi:10.1002/ehf2.12958

Karki, R., Sharma, B. R., Tuladhar, S., Williams, E. P., Zalduondo, L., Samir, P., et al. (2021). Synergism of TNF- $\alpha$ and IFN- $\gamma$ Triggers Inflammatory Cell Death, Tissue Damage, and Mortality in SARS-CoV-2 Infection and Cytokine Shock Syndromes. Cell 184 (1), 149-168. e117. doi:10.1016/ j.cell.2020.11.025

Kawai, T., and Akira, S. (2010). The Role of Pattern-Recognition Receptors in Innate Immunity: Update on Toll-like Receptors. Nat. Immunol. 11 (5), 373-384. doi:10.1038/ni.1863 
Kidane, T. Z., Sauble, E., and Linder, M. C. (2006). Release of Iron from Ferritin Requires Lysosomal Activity. Am. J. Physiology-Cell Physiol. 291 (3), C445-C455. doi:10.1152/ajpcell.00505.2005

Le Lan, C., Lore'al, O., Cohen, T., Ropert, M., Glickstein, H., Laine', F., et al. (2005). Redox Active Plasma Iron in C282Y/C282Y Hemochromatosis. Blood 105 (11), 4527-4531. doi:10.1182/blood-2004-09-3468

Mancias, J. D., Pontano Vaites, L., Nissim, S., Biancur, D. E., Kim, A. J., Wang, X., et al. (2015). Ferritinophagy via NCOA4 Is Required for Erythropoiesis and Is Regulated by Iron Dependent HERC2-Mediated Proteolysis. Elife 4, e10308. doi:10.7554/eLife.10308

Mancias, J. D., Wang, X., Gygi, S. P., Harper, J. W., and Kimmelman, A. C. (2014). Quantitative Proteomics Identifies NCOA4 as the Cargo Receptor Mediating Ferritinophagy. Nature 509 (7498), 105-109. doi:10.1038/nature13148

Mehta, P., McAuley, D. F., Brown, M., Sanchez, E., Tattersall, R. S., Manson, J. J., et al. (2020). COVID-19: Consider Cytokine Storm Syndromes and Immunosuppression. The Lancet 395 (10229), 1033-1034. doi:10.1016/ s0140-6736(20)30628-0

Oexle, H., Gnaiger, E., and Weiss, G. (1999). Iron-dependent Changes in Cellular Energy Metabolism: Influence on Citric Acid Cycle and Oxidative Phosphorylation. Biochim. Biophys. Acta (Bba) - Bioenerg. 1413 (3), 99-107. doi:10.1016/s0005-2728(99)00088-2

Perricone, C., Bartoloni, E., Bursi, R., Cafaro, G., Guidelli, G. M., Shoenfeld, Y., et al. (2020). COVID-19 as Part of the Hyperferritinemic Syndromes: the Role of Iron Depletion Therapy. Immunol. Res. 68 (4), 213-224. doi:10.1007/s12026020-09145-5

Philpott, C. C. (2018). The Flux of Iron through Ferritin in Erythrocyte Development. Curr. Opin. Hematol. 25 (3), 183-188. doi:10.1097/ moh.0000000000000417

Pretorius, E., and Kell, D. B. (2014). Diagnostic Morphology: Biophysical Indicators for Iron-Driven Inflammatory Diseases. Integr. Biol. 6 (5), 486-510. doi:10.1039/c4ib00025k

Rainey, N. E., Moustapha, A., Saric, A., Nicolas, G., Sureau, F., and Petit, P. X. (2019). Iron Chelation by Curcumin Suppresses Both Curcumin-Induced Autophagy and Cell Death Together with Iron Overload Neoplastic Transformation. Cell Death Discov. 5, 150. doi:10.1038/s41420-019-0234-y

Recalcati, S., Invernizzi, P., Arosio, P., and Cairo, G. (2008). New Functions for an Iron Storage Protein: The Role of Ferritin in Immunity and Autoimmunity. J. Autoimmun. 30 (1-2), 84-89. doi:10.1016/j.jaut.2007.11.003

Rosário, C., Zandman-Goddard, G., Meyron-Holtz, E. G., D'Cruz, D. P., and Shoenfeld, Y. (2013). The Hyperferritinemic Syndrome: Macrophage Activation Syndrome, Still's Disease, Septic Shock and Catastrophic Antiphospholipid Syndrome. BMC Med. 11, 185. doi:10.1186/1741-7015-11-185

Ruddell, R. G., Hoang-Le, D., Barwood, J. M., Rutherford, P. S., Piva, T. J., Watters, D. J., et al. (2009). Ferritin Functions as a Proinflammatory Cytokine via Ironindependent Protein Kinase C Zeta/Nuclear Factor KappaB-Regulated
Signaling in Rat Hepatic Stellate Cells. Hepatology 49 (3), 887-900. doi: $10.1002 /$ hep. 22716

Ruscitti, P., Cipriani, P., Ciccia, F., Di Benedetto, P., Liakouli, V., Berardicurti, O., et al. (2016). H-ferritin and CD68+/H-Ferritin+ Monocytes/macrophages Are Increased in the Skin of Adult-Onset Still's Disease Patients and Correlate with the Multi-Visceral Involvement of the Disease. Clin. Exp. Immunol. 186 (1), 30-38. doi:10.1111/cei.12826

Shoenfeld, Y. (2020). Corona (COVID-19) Time Musings: Our Involvement in COVID-19 Pathogenesis, Diagnosis, Treatment and Vaccine Planning. Autoimmun. Rev. 19 (6), 102538. doi:10.1016/j.autrev.2020.102538

Siddiqi, H. K., and Mehra, M. R. (2020). COVID-19 Illness in Native and Immunosuppressed States: A Clinical-Therapeutic Staging Proposal. J. Heart Lung Transplant. 39 (5), 405-407. doi:10.1016/j.healun.2020.03.012

Stockwell, B. R., Jiang, X., and Gu, W. (2020). Emerging Mechanisms and Disease Relevance of Ferroptosis. Trends Cel Biol. 30 (6), 478-490. doi:10.1016/ j.tcb.2020.02.009

Tang, M., Chen, Z., Wu, D., and Chen, L. (2018). Ferritinophagy/ferroptosis: Ironrelated Newcomers in Human Diseases. J. Cel Physiol 233 (12), 9179-9190. doi:10.1002/jcp.26954

Yang, X., Yu, Y., Xu, J., Shu, H., Xia, J. A., Liu, H., et al. (2020). Clinical Course and Outcomes of Critically Ill Patients with SARS-CoV-2 Pneumonia in Wuhan, China: a Single-Centered, Retrospective, Observational Study. Lancet Respir. Med. 8 (5), 475-481. doi:10.1016/s2213-2600(20)30079-5

Zhang, W., Zhao, Y., Zhang, F., Wang, Q., Li, T., Liu, Z., et al. (2020). The Use of Anti-inflammatory Drugs in the Treatment of People with Severe Coronavirus Disease 2019 (COVID-19): The Perspectives of Clinical Immunologists from China. Clin. Immunol. 214, 108393. doi:10.1016/ j.clim.2020.108393

Conflict of Interest: The authors declare that the research was conducted in the absence of any commercial or financial relationships that could be construed as a potential conflict of interest.

Publisher's Note: All claims expressed in this article are solely those of the authors and do not necessarily represent those of their affiliated organizations, or those of the publisher, the editors, and the reviewers. Any product that may be evaluated in this article, or claim that may be made by its manufacturer, is not guaranteed or endorsed by the publisher.

Copyright (c) $2021 \mathrm{Jia}$, Liu and Kang. This is an open-access article distributed under the terms of the Creative Commons Attribution License (CC BY). The use, distribution or reproduction in other forums is permitted, provided the original author(s) and the copyright owner(s) are credited and that the original publication in this journal is cited, in accordance with accepted academic practice. No use, distribution or reproduction is permitted which does not comply with these terms. 